\title{
EXPLORING NATURAL VARIATION OF ROOT ARCHITECTURAL TRAITS IN SEEDLINGS OF MAIZE HYBRID AND INBRED LINES FROM THAILAND
}

\author{
SAlungyu, J. ${ }^{1}-$ KengKanna, J. ${ }^{1}-$ LAVOY, W. ${ }^{1}-$ SAEngWILAI, P. ${ }^{1,2^{*}}$ \\ ${ }^{I}$ Department of Biology, Faculty of Science, Mahidol University \\ Bangkok 10400, Thailand \\ ${ }^{2}$ Center of Excellence on Environmental Health and Toxicology (EHT), Faculty of Science, \\ Mahidol University, Bangkok 10400, Thailand \\ *Corresponding author \\ e-mail: patompong.sae@mahidol.edu \\ (Received $18^{\text {th }}$ Sep 2017; accepted $12^{\text {th }}$ Jan 2018)
}

\begin{abstract}
Root architectural traits refer to a spatial configuration of the root system providing a critical role in soil resource acquisition. In past decades, several lines of evidence have shown that maize possesses large phenotypic variations, including root architecture closely relating to physiological utility. Thailand has developed a unique maize germplasm over the years; however, root traits have often been neglected and have yet to be explored. In this present study, we examined natural variation of root architectural traits of twenty-eight inbred and hybrid lines at the seedling stage using a well-established roll-up culture system. We found that variation in root traits ranged from 4.22-fold in primary roots to 13.14-fold in seminal root length, while shoot mass presented the highest phenotypic variation among traits (23.42-fold). Most root architectural traits of maize hybrid lines were significantly higher than the inbred lines, particularly, seminal root length, which was $34.30 \%$ greater than that of inbred lines. In addition, we found that root traits, like other agronomic traits, express heterosis. Our findings suggest ample opportunity for incorporating root traits in maize breeding program in Thailand.
\end{abstract}

Keywords: maize, hybrid, breeding, seminal roots, roll-up

\section{Introduction}

Maize (Zea mays L.) is the world's most widely grown crop and is used as a staple food source, especially in Latin America and Africa. It has been estimated that within a decade, the global demand for maize will surpass other cereal crops (Ranum et al., 2014; Reynolds et al., 2015). This poses a challenge to maize breeders and farmers who must increase maize production. While developing nations are struggling with limited resources and inadequate accessibility to technologies, rich nations have applied excessive amount of fertilizers leading to substantial pollution of air and water resources (Tilman et al., 2002). In Thailand, maize is one of five prominent crops grown in the highlands. The Thai government has long supported the expansion of maize farming areas and the improvement of varieties to withstand various environmental conditions (Ekasingh et al., 2014). The 3 most popular hybrid maize varieties cultured in Thailand are Nakhon Sawan 3, NSX 052014 and NSX 111044 (Butthong et al., 2014). These genotypes produce high yields and are able to tolerate drought conditions (Butthong et al., 2014). Nevertheless, Thai maize production was reduced by $17.69 \%$ in the past decade (Pipitkul et al., 2015). The reductions in maize yield is primarily attributed to drought (Pipitkul et al., 2015). Not only is the increase in maize production 
necessary, but it will require substantial changes in agronomic practices and methods for crop improvement.

Plant root system plays important role in plant growth, resource allocation, and acquisition of water and nutrients from soil. Therefore, improving plant roots could lead to increased crop productivity (Lynch, 2007). The maize root system consists of two components: the embryonic and post embryonic root system. The embryonic root system is formed during embryogenesis and it is composed of a primary root and a variable number of seminal roots. The postembryonic root system is formed during postembryonic development and it is composed of shoot borne roots and lateral roots that originate in the pericycle of other root types. Shoot borne roots formed below the soil level are referred to as crown roots, while those formed aboveground are designated as brace roots (Bennetzen et al., 2009). Identification of relevant root traits is essential for plant breeders in crop improvement programs. The goals of root breeding are to help soil exploration and effective use of water and nutrients, while also maintaining growth and crop productivity. Lynch (2013) proposed an ideotype of maize root systems for higher acquisition of water and nitrogen called "Steep, Cheap, and Deep". A primary root with a large diameter and few long lateral roots was proposed as ideal characteristics for deeper rooting and greater access to water and nitrate residing deep in the soil strata under drought and low nitrogen conditions (Lynch, 2013). Crown root system with a low amount of crown root and few long lateral roots is suggested as the primary trait for enhancing water acquisition. Moreover, seminal roots with shallow root angles, a small diameter, high lateral root density and long root hairs are suitable for phosphorus and potassium uptake, while long and low lateral root density are optimal for nitrogen and water acquisition (Postma et al., 2014). Anatomical traits also play important roles in improving water and nitrogen use efficiency in maize. Root cortical aerenchyma (RCA), tissues with large intercellular spaces, reduces root respiration and allows deeper rooting in the subsoil (Zhu et al., 2010). Several studies confirmed that high RCA maize varieties are able to tolerate and improve their growth under stressful conditions such as low nitrogen soil and drought (Zhu et al., 2010; Saengwilai et al., 2014a).

For crop selection and plant breeding purposes, the evaluation of root traits of a large number of mature plants in the field may not always be practical. Since seedlings with beneficial root traits could subsequently develop a more vigorous root system, resulting in more biomass and yield (Bocev, 1963), researchers are turning toward identification of root characteristics in plant seedlings that are associated with adaptation of mature plants in the field. For examples, Thomas et al. (2016) demonstrated that Brassica napus with long primary root length identified in 2-week-old seedlings in a pouch system was correlated with shoot mass $(\mathrm{r}=0.49, \mathrm{p}<0.001)$ and yield $(\mathrm{r}=0.50$, $\mathrm{p}<0.01$ ) of plants in the field (Thomas et al., 2016). In maize, Tuberosa et al. (2002) showed that there were significant negative correlations between seminal root weight and primary root diameter of 21-day-old seedlings grown in hydroponics and grain yield in mature plants in the field under drought conditions (Tuberosa et al., 2002). In addition, root traits measured at the seedling stage such as primary root length, number of crown roots, number of seminal roots, number of lateral roots, fresh root weight, and dry root weight were shown to have high heritability under normal and drought conditions and thus seedling root traits can be improved through selection and breeding (Qayyum et al., 2012). 
Maize breeders often develop hybrids to benefit from heterosis, which is defined as the superior performance of an F1 hybrid compared with the performance of the inbred parents (Hoecker et al., 2006; Bennetzen et al., 2009). The phenomenon of heterosis in maize has been well described not only for shoot traits and yield, but also for root traits including primary root length, primary root width, lateral root density and longitudinal size of cortical cell (Hoecker et al., 2006). For example, F1 hybrids developed from a cross between UH250xUH301 had $110 \%$ greater lateral root density compared to their parents at 5 days after germination (DAG) while seminal root number of hybrids were $55.56 \%$ greater than the parents at 14 DAG (Hoecker et al., 2006). To benefit from heterosis, breeders combine the heterotic groups while inbred lines are kept and selected separately within these groups. In Thailand, there is a lack of knowledge on root traits of inbred and hybrid maize lines currently used in the breeding program, therefore, the information of phenotypic variation and heterosis of root traits are required for the development of breeding program targeting root traits. In this study, we aimed (1) to explore phenotypic variation of root architectural traits among twenty-eight Thai maize lines during seedling stage. We also (2) compared root architectural traits between Thai maize inbred and hybrid lines, and (3) investigated possible correlations among root architectural traits.

\section{Materials and methods}

\section{Plant materials}

Twenty-eight Thai maize lines were used to perform the experiment. These lines consisted of 6 inbred lines from the National Research Centre of Millet and Corn (Suwan Farm), and 12 inbred and 10 hybrid lines from Nakhon Sawan Field Crops Research Center (NSFCRC). Among the hybrid lines, NS2 is derived from a cross between Tak Fa 1 and 2 while NS3 is from Tak Fa1 and 3 (Table 1).

\section{Roll-up paper culture system}

The roll-up paper culture system was prepared according to the protocol described by Zhu et al. (2006). Specifically, seeds were sterilized in 10\% (v/v) $\mathrm{NaOCl}$ for $1 \mathrm{~min}$ followed by soaking with distilled water. The experiment was a randomized complete block design with four replicates. In each replicate, five seeds of uniform size for each maize line were selected and were wrapped in brown germination paper as a cigar roll. The roll-up papers were soaked vertically with $0.5 \mathrm{mM} \mathrm{CaSO}_{4}$ in beakers and placed in darkness at $28 \pm 1^{\circ} \mathrm{C}$ for 3 days (Saengwilai et al., 2014b). The seedlings were then grown under a photoperiod of 12/12 h with photosynthetically active radiation of 200 $\mu \mathrm{mol}$ photo $\mathrm{m}^{-2} \mathrm{~s}^{-1}$. Roots and shoots were evaluated at 7 days after germinating.

\section{Root trait measurement}

At harvest, two plants per line in each replication were selected and then shoots were separated from the roots. To evaluate root architectural traits including primary root length, seminal root number and seminal root length, the roots were scanned by using Epson Perfection V800 Photo Color Scanner (Epson America, Inc. Company). The root images were analyzed by ImageJ program (Schindelin et al., 2013). Lateral root numbers were counted using ImageJ program, while lateral root density was determined by calculating lateral root number per 1 centimetre of primary root length. Moreover, 
both shoots and roots from each line were dried at $60{ }^{\circ} \mathrm{C}$ for $48 \mathrm{~h}$ and then weighed by microbalance.

\section{Statistical analysis}

Statistical analyses were performed using package R, version 3.4.1 (R Core Team, 2017). One and Two-way ANOVA was used to evaluate phenotypic differences within the inbred and hybrid lines and between these two groups for all traits. Tukey's Honest Significant Difference (HSD) test $(\alpha=0.05)$ was used for multiple comparison tests. A correlation analysis was performed on corresponding traits using the mean values of the traits. A principal component analysis (PCA) was performed within each group. The first two components were characterized based on variable eigenvalues and on vector clustering within plots of components 1 and 2 .

\section{Results}

\section{Phenotypic variation of Thai maize root traits in seedling stage}

Twenty-eight Thai maize lines were planted in a roll-up system to investigate root architectural traits during the seedling stage (Fig. 1). Maize lines were grouped based on individual descent, composing of 18 inbred and 10 hybrid lines. The range of all of the values was examined to determine the variation of each root architectural trait. Significant variations of root architectural traits are presented in Table 1. Among root traits, primary root length had the lowest range of variation, while seminal root length had the greatest variation. However, when comparing ranges among all traits, shoot mass had the highest phenotypic variation of 23.42-fold difference.

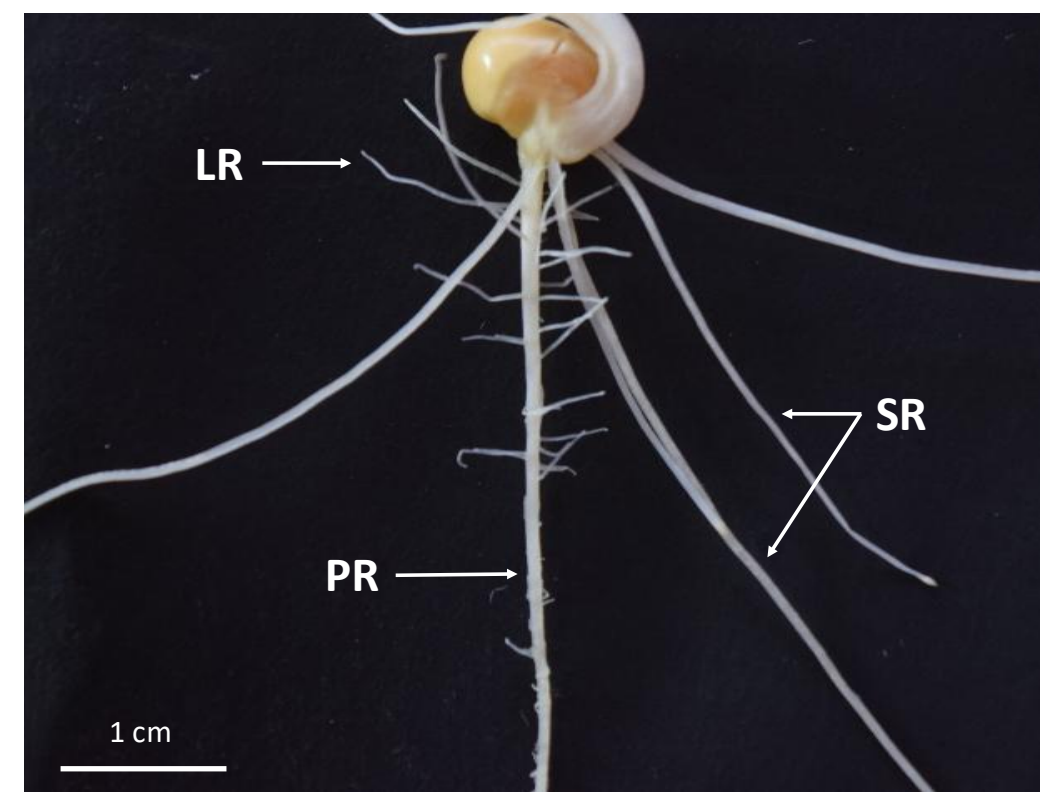

Figure 1. Root system of a 7-day-old maize seedling showing a primary root (PR), seminal roots $(S R)$, and lateral roots $(L R)$

SW14D-C5 1039-60 Ki60 appeared to have higher overall values of root architectural traits such as total seminal root number and lateral root density among inbred lines 
(Table 1). This line also had the greatest shoot mass and dry mass compared to other inbred lines. Among hybrid lines, NSX 102005 had the highest primary root length $(21.74 \pm 0.62 \mathrm{~cm})$, while NSX 042022 had the highest total seminal root number $(5.38 \pm$ $0.18)$, lateral root density $(5.92 \pm 0.58$ roots $/ 1 \mathrm{~cm}$ of primary root $)$ and shoot mass $(0.077 \pm 0.040 \mathrm{~g})$. Comparing root traits of NS3 with its parents Tak Fa 1 and Tak Fa 3, we found that most of root architectural traits express heterosis except root mass which was not significant among the lines (Table 1).

Table 1. Phenotypic variation of Thai maize root traits in seedling stage. Root traits including PRL; Primary root length (cm), TSN; Total seminal root number (roots), SRL; Seminal root length (cm) and LRD; Lateral root density (roots per $1 \mathrm{~cm}$ of primary root) were quantified. SM; Shoot mass (g) and Root mass (g) were also investigated

\begin{tabular}{|c|c|c|c|c|c|c|c|}
\hline \multirow{2}{*}{ Lines } & \multicolumn{6}{|c|}{ Root traits } & \multirow{2}{*}{ Type } \\
\hline & PRL & TSN & SRL & LRD & $\mathbf{R M}$ & SM & \\
\hline SW14D-C5 1039-3 Ki3 & $16.96 \pm 0.89$ & $3.13 \pm 0.40$ & $8.90 \pm 0.39$ & $1.71 \pm 0.26$ & $0.15 \pm 12.07$ & $0.026 \pm 0.002$ & Inbred \\
\hline SW14D-C5 1039-11 Ki11 & $12.80 \pm 1.17$ & $3.38 \pm 0.46$ & $8.89 \pm 0.84$ & $3.25 \pm 0.38$ & $0.15 \pm 12.34$ & $0.032 \pm 0.003$ & Inbred \\
\hline SW14D-C5 1039-21 Ki21 & $17.66 \pm 0.89$ & $3.50 \pm 0.19$ & $15.05 \pm 0.96$ & $4.13 \pm 0.89$ & $0.23 \pm 9.13$ & $0.031 \pm 0.002$ & Inbred \\
\hline SW14D-C5 1039-48 Ki48 & $20.26 \pm 0.70$ & $4.88 \pm 0.23$ & $14.89 \pm 0.59$ & $3.25 \pm 0.33$ & $0.16 \pm 11.38$ & $0.030 \pm 0.002$ & Inbred \\
\hline SW15D-B5 1006-20 Ki53 & $17.59 \pm 0.70$ & $3.38 \pm 0.32$ & $8.82 \pm 0.67$ & $4.67 \pm 0.58$ & $0.24 \pm 9.05$ & $0.026 \pm 0.001$ & Inbred \\
\hline SW14D-C5 1039-60 Ki60 & $17.75 \pm 1.40$ & $5.13 \pm 0.35$ & $13.95 \pm 0.87$ & $6.08 \pm 0.29$ & $0.25 \pm 8.76$ & $0.037 \pm 0.002$ & Inbred \\
\hline Nei 452006 & $17.21 \pm 1.00$ & $4.25 \pm 0.25$ & $10.71 \pm 0.86$ & $5.29 \pm 0.39$ & $0.15 \pm 12.00$ & $0.030 \pm 0.001$ & Inbred \\
\hline Nei 452007-1 & $15.91 \pm 0.85$ & $3.75 \pm 0.37$ & $11.73 \pm 0.83$ & $4.58 \pm 0.80$ & $0.13 \pm 13.48$ & $0.025 \pm 0.002$ & Inbred \\
\hline Nei 452009 & $15.04 \pm 0.72$ & $3.63 \pm 0.32$ & $9.29 \pm 0.79$ & $3.21 \pm 0.42$ & $0.14 \pm 9.60$ & $0.035 \pm 0.003$ & Inbred \\
\hline Nei 462013 & $15.14 \pm 1.44$ & $3.88 \pm 0.30$ & $9.59 \pm 1.28$ & $0.79 \pm 0.21$ & $0.18 \pm 10.76$ & $0.026 \pm 0.001$ & Inbred \\
\hline Nei 532005 & $16.78 \pm 1.35$ & $3.00 \pm 0.33$ & $7.51 \pm 0.51$ & $2.92 \pm 0.61$ & $0.17 \pm 10.93$ & $0.025 \pm 0.002$ & Inbred \\
\hline Nei 542011 & $16.35 \pm 0.85$ & $1.88 \pm 0.35$ & $8.18 \pm 1.62$ & $3.79 \pm 0.19$ & $0.17 \pm 11.29$ & $0.024 \pm 0.002$ & Inbred \\
\hline Nei 542014 & $15.15 \pm 0.30$ & $3.00 \pm 0$ & $11.93 \pm 0.86$ & $4.17 \pm 0.57$ & $0.14 \pm 12.55$ & $0.027 \pm 0.001$ & Inbred \\
\hline Nei 542017 & $15.14 \pm 1.25$ & $3.38 \pm 0.32$ & $13.56 \pm 0.55$ & $6.08 \pm 0.30$ & $0.14 \pm 12.72$ & $0.025 \pm 0.001$ & Inbred \\
\hline Nei 542019 & $15.87 \pm 1.50$ & $3.13 \pm 0.23$ & $13.86 \pm 0.89$ & $3.92 \pm 0.68$ & $0.13 \pm 13.15$ & $0.023 \pm 0.001$ & Inbred \\
\hline Tak Fa 1 & $17.52 \pm 0.85$ & $4.00 \pm 0.27$ & $12.33 \pm 0.87$ & $5.33 \pm 0.41$ & $0.15 \pm 12.28$ & $0.031 \pm 0.002$ & Inbred \\
\hline Tak Fa 2 & $14.41 \pm 0.89$ & $1.25 \pm 0.53$ & $5.66 \pm 1.50$ & $3.29 \pm 0.65$ & $0.14 \pm 12.80$ & $0.019 \pm 0.003$ & Inbred \\
\hline Tak Fa 3 & $13.78 \pm 1.49$ & $4.63 \pm 0.32$ & $9.76 \pm 1.24$ & $2.58 \pm 0.43$ & $0.15 \pm 11.98$ & $0.034 \pm 0.002$ & Inbred \\
\hline NSX 042007 & $21.11 \pm 0.50$ & $5.00 \pm 0.46$ & $16.76 \pm 0.85$ & $4.63 \pm 0.30$ & $0.14 \pm 12.84$ & $0.036 \pm 0.002$ & Hybrid \\
\hline NSX 042022 & $20.48 \pm 0.51$ & $5.38 \pm 0.18$ & $15.96 \pm 0.71$ & $5.92 \pm 0.58$ & $0.13 \pm 13.07$ & $0.077 \pm 0.040$ & Hybrid \\
\hline NSX 052014 & $21.22 \pm 0.77$ & $5.38 \pm 0.26$ & $18.33 \pm 0.93$ & $3.38 \pm 0.15$ & $0.21 \pm 9.78$ & $0.046 \pm 0.002$ & Hybrid \\
\hline NSX 102005 & $21.74 \pm 0.62$ & $4.75 \pm 0.41$ & $17.51 \pm 1.2$ & $5.92 \pm 0.25$ & $0.16 \pm 11.71$ & $0.041 \pm 0.004$ & Hybrid \\
\hline NSX 112011 & $13.78 \pm 0.47$ & $4.71 \pm 0.89$ & $11.97 \pm 0.82$ & $2.13 \pm 0.43$ & $0.17 \pm 8.50$ & $0.027 \pm 0$ & Hybrid \\
\hline NSK 112014 & $21.29 \pm 0.34$ & $5.13 \pm 0.23$ & $17.40 \pm 0.84$ & $4.83 \pm 0.60$ & $0.19 \pm 10.4$ & $0.050 \pm 0.002$ & Hybrid \\
\hline NSX 112017 & $20.84 \pm 0.58$ & $4.50 \pm 0.19$ & $17.49 \pm 1.00$ & $5.92 \pm 0.42$ & $0.14 \pm 12.83$ & $0.032 \pm 0.002$ & Hybrid \\
\hline NSX 112019 & $21.69 \pm 0.39$ & $4.75 \pm 0.25$ & $20.54 \pm 0.45$ & $4.83 \pm 0.36$ & $0.11 \pm 14.78$ & $0.037 \pm 0.001$ & Hybrid \\
\hline NS 2 & $21.25 \pm 0.75$ & $4.00 \pm 0.45$ & $9.99 \pm 3.79$ & $3.83 \pm 1.50$ & $0.18 \pm 8.08$ & $0.022 \pm 0.014$ & Hybrid \\
\hline NS 3 & $22.29 \pm 0.67$ & $5.25 \pm 0.37$ & $18.03 \pm 1.22$ & $5.50 \pm 0.39$ & $0.14 \pm 12.64$ & $0.050 \pm 0.001$ & Hybrid \\
\hline Mean & 17.71 & 4 & 12.93 & 4.2 & 0.16 & 0.03 & \\
\hline Standard error & 0.25 & 0.092 & 0.315 & 0.124 & 0.002 & 0.001 & \\
\hline Range & 4.22 & 9 & 13.14 & 9 & 3.65 & 23.42 & \\
\hline F-value of variability & $9.198 * * *$ & $8.793 * * *$ & $13.170 * * *$ & $8.256^{* * *}$ & $6.319 * * *$ & $2.100 * *$ & \\
\hline Degree of freedom & 27 & 27 & 27 & 27 & 27 & 27 & \\
\hline Mean square & 63.42 & 8.641 & 116.49 & 15.04 & 0.005 & 0.001 & \\
\hline
\end{tabular}

$* * *, * * *$ indicated significant level at $\mathrm{p}<0.001, \mathrm{p}<0.01, \mathrm{p}<0.05$. All traits varied significantly within each population 


\section{Comparing root and shoot traits between maize inbred and hybrid lines}

We found that root traits of hybrid lines are significantly different from those of inbred lines (Fig. 2). Primary root length of hybrid lines was $22.18 \%$ greater than that of inbred lines. This trend remained consistent with seminal root traits, lateral root traits and shoot mass. Hybrid lines had greater seminal root length and seminal root number by $34.30 \%$ and $28.56 \%$ respectively compared to inbred lines. Lateral root density of maize hybrid lines was $21.20 \%$ higher when compared with inbred lines. In addition, shoot mass of hybrid lines was greater than inbred lines by $33.65 \%$. Interestingly, an average root mass of hybrid lines was not significantly different from inbred varieties.
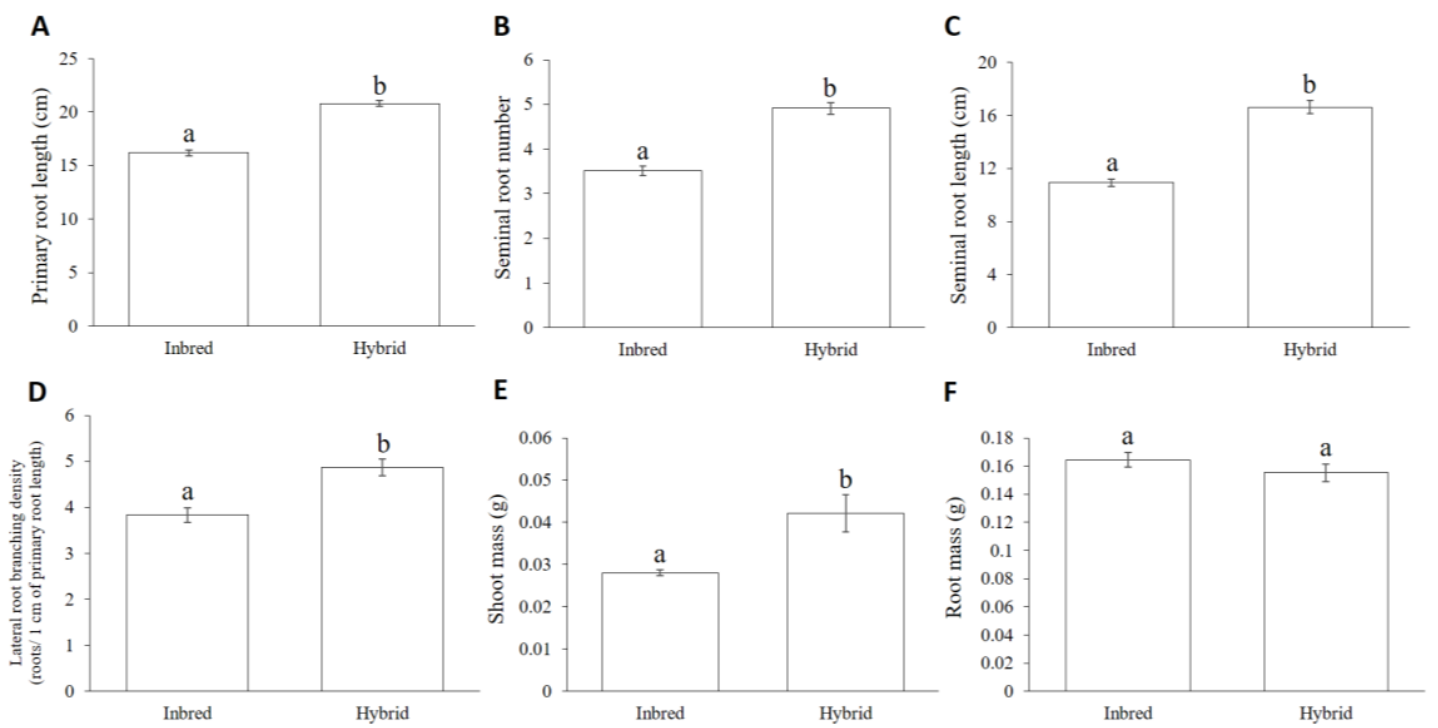

Figure 2. Phenotypic variation of Thai maize root traits in seedling stage comparing between inbred and hybrid lines using Tukey's HSD. Root traits including PRL; Primary root length, TSN; Total seminal root number, SRL; Seminal root length and LRD; Lateral root density were quantified. SM; Shoot mass and Root mass were also investigated. The data shown are means of four replicates $\pm S E$. Different letters represent significant differences $(P<0.05)$. All traits varied significantly within each group

\section{Correlations among root traits and principal component analysis}

We further investigated correlations among traits. We found that primary root length was highly correlated with seminal and lateral root traits. As shown in Table 2, primary root length correlated with seminal root length $\left(\mathrm{r}^{2}=0.74\right)$. Additionally, primary root length had a significant positive correlation with shoot mass $\left(r^{2}=0.52\right)$. Within the seminal root class, seminal root length correlated with total seminal root number $\left(r^{2}=0.47\right)$ and lateral root density on seminal roots $\left(r^{2}=0.42\right)$. Interestingly, seminal root length highly correlated with shoot mass $\left(\mathrm{r}^{2}=0.58\right)$.

Principal component analysis (PCA) of root architectural traits and root biomass were performed separately on Thai maize inbred and hybrid lines. For inbred lines, principal component 1 (PC1) and PC2 contributed $67.3 \%$ of the total variation in plant traits (Fig. 3A). While, the first two components of hybrid lines accounted for $74.7 \%$ of the total variation (Fig. 3B). Root architectural traits, such as primary root length, seminal root length, seminal root number, and lateral root density of inbred lines 
strongly associated with PC1. Similarly, primary root length, seminal root length, and lateral root density of hybrid lines were also heavily involved in PC1 (Fig. 3B). Seminal root length appeared to be a major contributor to PC1 in both inbred and hybrid group. While, seminal root number was a major contributor to PC2 in hybrid lines, and root mass contributing heavily to PC2 among maize inbred lines.

Table 2. Correlation of root and physiological traits of seedling among 28 Thai maize lines at 7 DAP in roll-up system. PRL; Primary root length, TSN; Total seminal root number, SRL; Seminal root length and LRD; Lateral root density, SM; Shoot mass and Root mass were also investigated

\begin{tabular}{c|c|c|c|c|c}
\hline TSN & $0.43^{* * *}$ & & & & \\
SRL & $0.74^{* * *}$ & $0.47^{* * *}$ & & & \\
LRD & $0.39^{* * *}$ & $0.22^{* *}$ & $0.42^{* * *}$ & & \\
SM & $0.52^{* * *}$ & $0.48^{* * *}$ & $0.58^{* * *}$ & $0.30^{* *}$ & \\
RM & -0.074 & -0.04 & -0.13 & $-0.16^{*}$ & -0.019 \\
\hline & PRL & TSN & SRL & LRD & SM \\
\hline
\end{tabular}

$* * *, * *, *$ indicated significant level at $\mathrm{p}<0.001, \mathrm{p}<0.01, \mathrm{p}<0.05$ as Spearman's principle correlation. All traits varied significantly within each population
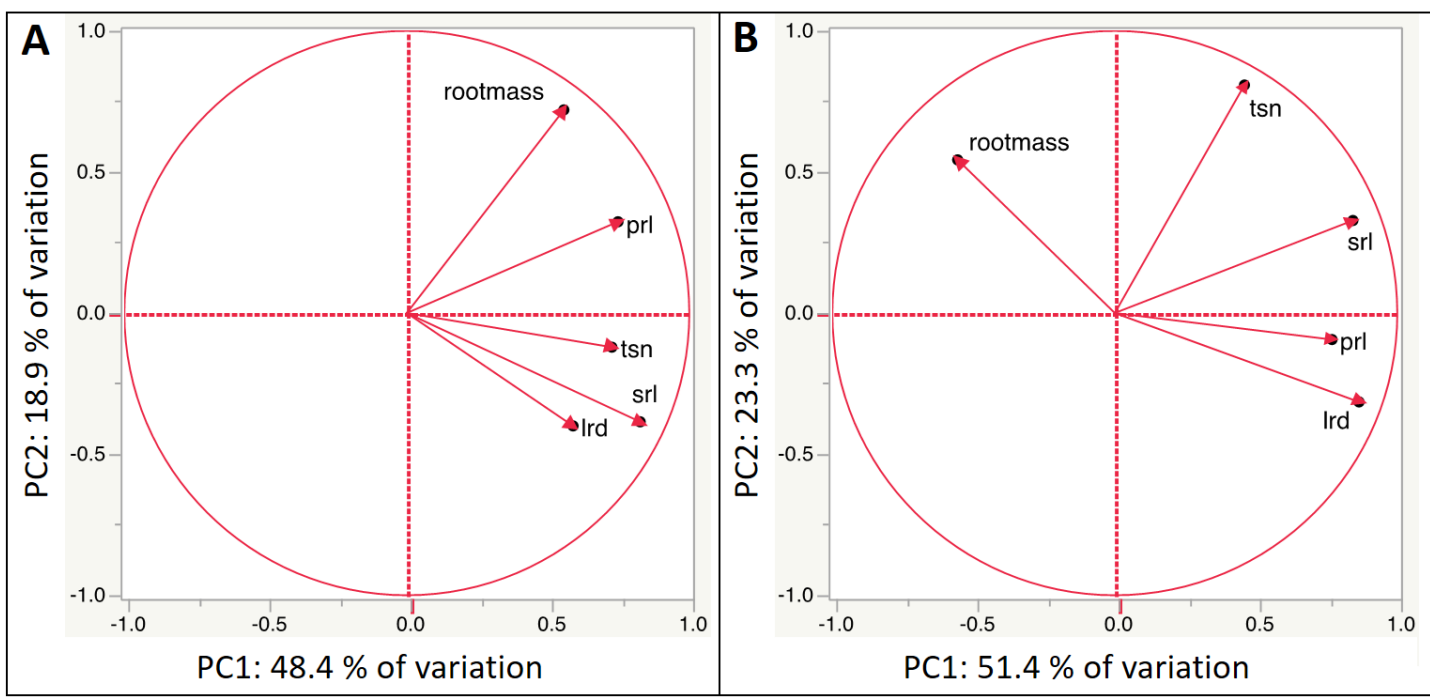

Figure 3. Biplots of principal component 1 and 2 of root architectural traits and root biomass in Thai maize inbred (A) and hybrid lines (B). The $x$ and y axes are components 1 and 2 , respectively. Axis labels include the percentage of variation explained by each of these two components

\section{Discussion}

From the beginning of maize domestication shoot traits have been the main focus for crop improvement. The below-ground part, roots, have often been neglected or indirectly selected despite their importance for water and nutrient acquisition. During the past decades, several lines of evidence have shown that variation in root traits largely influences adaptation of maize in the field. (Hufford et al., 2012; York et al., 2015). Therefore, root traits have increasingly become a target for maize improvement 
worldwide. The results of the present study show that Thai maize lines exhibit a large natural variation of root traits relating to their abilities in water and nutrient uptake.

Root system of plant seedlings play an important role in plant establishment and thus influence the likelihood of survival to reproduction (Tuberosa et al., 2009). The variation of root number, length, and branching of seedling roots have been shown to be associated with maize adaptation to edaphic stresses. In our study, we found that maize lines were significantly different in their root traits, particularly, seminal roots. The range of seminal root length found among hybrid Thai maize lines (9.9-20.5) fell within the same range as those from seventy-five B73 $\times$ Gaspé Flint introgression library (8.829.3) grown in the same roll up system (Salvi et al., 2016). Interestingly, Thai maize had greater range of seminal root number (4.0-5.4) compared to the 75 hybrid lines (0.23.7) studied by Salvi et al. (2016). Seminal root traits can vary depending on genetic background of each line (Hochholdinger et al., 2004a; Hochholdinger et al., 2004b; Hochholdinger et al., 2005). Environmental factors may increase seminal root number especially when nutrient limitation such as phosphorus occurs (Zhu et al., 2006). Having high plasticity in both length and number in response to the environment, seminal roots play a large role in soil exploration, and therefore, are critical for plant's establishment and could serve as a great target trait for maize improvement (Richards, 2006; Zhu et al., 2006; Lynch, 2013).

Correlation analysis indicated that most root traits positively correlated amongst each other, with root mass as an exception only significantly correlating with lateral root density. Previous studies have already shown that an enlarged root system through the alteration of root length, density and root mass is beneficial for nitrogen and water acquisition (Ehdaie et al., 2010; Palta et al., 2011). Root mass is an integrated trait which reflects plant's carbon investment to the whole root structure. In terms of carbon investment, having a large root mass may be detrimental to plants under drought or suboptimal nutrient conditions. The competition between roots and shoots for photosynthates may result in overall reduction in growth rate and yield. The formation of large root systems incurs a high carbon cost. To survive in a depriving environment, genotypes with root traits enhancing soil exploration with minimal carbon investment are the most competitive (Lynch, 2005). Among maize root architectural traits, lateral roots are considered carbon efficient because they are smaller in diameter, have lower carbon investment than main axis roots, and are crucial for nutrient exploration in both topsoil and subsoil layers (Lynch, 2013). We found that plants with low root weight tended to have dense lateral root density such as Nei 452007-1and Nei 542017. Compared to primary and seminal roots which are axial roots determined by genetic inheritance, lateral roots are the only root class that can be formed at the pericycle layer in every root class, and respond to unfavourable environments such as phosphorus deficiency (Lynch et al., 2005; Bennetzen et al., 2009). Therefore, carbon resources of the plant might be translocated to optimize nutrient acquisition by altering their lateral root numbers.

We found that hybrid maize had greater root length and number than inbred maize. This is consistent with other studies (Hoecker et al., 2006; Chairi et al., 2016). With a detailed observation, we found that root traits, like other agronomic traits, express heterosis as evidenced by the observation of root traits of NS3 and its parents, Tak Fa 1 and Tak Fa 3. Heterosis refers to the performance of the hybrid F1 generation that exhibits phenotypic traits above the average of the two parents in regards to viability, development, and production (Tollenaar et al., 2004; Chairi et al., 2016). Heterosis of 
hybrid lines has been shown to improve agronomic traits of maize (Springer et al., 2007; Garcia et al., 2008; Liu et al., 2013; Thiemann et al., 2014). For example, F1 hybrids developed from a cross between B73 and Mo17, had greater ear length, height, seed weight (g/ear), and 11 DAP seedling biomass by $48.3 \%, 21.8 \%, 101.7 \%$, and $69.0 \%$ respectively (Auger et al., 2005). Additionally, the B73xMo17 hybrids further demonstrated heterosis with an increase of yields $(\mathrm{mg} / \mathrm{ha}$ ) by $64.7 \%$ (Zanoni et al., 1989). However, the quantitative measurements of heterosis varies significantly among traits and hybrid lines (Springer et al., 2007). Several studies have been carried out in order to attempt to explain the promising genetic principle of heterosis. The high vigor of hybrids over inbred lines has been shown to be contributed by many genes (Birchler et al., 2003; 2006; Hoecker et al., 2006; Liu et al., 2013). Among different root traits, root mass of NS3 did not show heterosis and was not significantly different from those of its parents. This result suggests despite being highly genetically dependant, breeding techniques that target root mass may be more complicated than initially thought. More research on heterosis of root traits is needed in order to successfully implement root traits of hybrid lines in a plant breeding program.

In the past decade, root traits of seedlings have been subjected to quantitative trait loci (QTL) studies and several significant QTL have been identified. For examples, Zhu et al. (2006) reported several QTLs for seminal root length and number of maize seedlings of the IBM population under high and low phosphorus conditions. The identified QTLs were on chromosomes 1, 2, 3 under high phosphorus and on chromosomes 1, 2, 6 under phosphorus deficiency (Zhu et al., 2006). Furthermore, Burton et al. (2014) reported 15 QTLs controlling length of multiple root classes, diameter and number of seminal roots, and dry weight of embryonic and nodal root systems of 28-day-old maize seedlings (Burton et al., 2014). The identification of QTLs associated with seedling root traits makes it possible to target root traits by marker assisted selection during breeding (Tuberosa et al., 2002; Hochholdinger et al., 2004b; Hochholdinger et al., 2008).

\section{Conclusions}

It has been shown that variation of root traits in maize influences water and nutrient acquisition under fertile and resource-limiting conditions (Chimungu et al., 2014a; 2014b; Saengwilai et al., 2014a; 2014b; Gao et al., 2016). This study demonstrates that Thai maize possesses phenotypic differences in root architectural traits and reveal potential donors for plant breeding targeting root traits such as SW14D-C5 1039-48 Ki48 for long primary and seminal root length and Nei 542017 for increased lateral root density. Since evaluation of root traits from mature field-grown plants are often laborious, time-consuming and destructive, screening for root traits in plant seedlings provides a more feasible option for breeders because a large number of genotypes can be evaluated in a relatively short period of time. Further studies are underway to investigate levels of plasticity among root traits in response to environmental stresses and transitioning of root traits from seedlings in the roll-up system to the field.

Acknowledgements. We thank Dr. Sansern Jampatong from National Research Centre of Millet and Corn (Suwan Farm) and and Mr. Suriphat Thaitad from Nakhon Sawan Field Crops Research Center (NSFCRC) for Thai maize lines seed supply and advice. This research project is supported by Mahidol University. 


\section{REFERENCES}

[1] Auger, D. L., Gray, A. D., Ream, T. S., Kato, A., Coe, E. H., Birchler, J. A. (2005): Non additive gene expression in diploid and triploid hybrids of maize. - Genetics 169: 389397.

[2] Bennetzen, J., Hake, S., Hochholdinger, F. (2009): Handbook of Maize: Its Biology. Springer Science, New York.

[3] Birchler, J. A., Auger, D. L., Riddle, N. C. (2003): In search of the molecular basis of heterosis. - The Plant Cell 15(10): 2236-2239.

[4] Birchler, J. A., Yao, H., Chudalayandi, S. (2006): Unraveling the genetic basis of hybrid vigor. - Proceedings of the National Academy of Sciences of the United States of America 103(35): 12957-12958.

[5] Bocev, B. (1963): Maize selection at an initial phase of development. - Crop Science 1(54): 179-182.

[6] Burton, A. L., Johnson, J. M., Foerster, J. M., Hirsch, C. N., Buell, C. R., Hanlon, M. T., Kaeppler, S. M., Brown, K. M., Lynch, J. P. (2014): QTL mapping and phenotypic variation for root architectural traits in maize (Zea mays L.). - Theoretical and Applied Genetics 127(11): 2293-2311.

[7] Butthong, T., Thaitad, S., Krutloima, P., Wongsupthai, S., Chaitawon, C., Tiampeng, P., Malipan, A., Seangkao, S., Praphet, A., Kanlayasillapin, P., Seangsoda, P. (2014): Regional yield trial: promising drought tolerance hybrid maize (early maturity). - Annual Report 13-24, Department of Agriculture, Ministry of Agriculture and Cooperatives, Thailand.

[8] Chairi, F., Elazab, A., Sanchez-Bragado, R., Araus, J. L., Serret, M. D. (2016): Heterosis for water status in maize seedlings. - Agricultural Water Management 164: 100-109.

[9] Chimungu, J. G., Brown, K. M., Lynch, J. P. (2014a): Large root cortical cell size improves drought. - Plant Physiology 166: 2166-2178.

[10] Chimungu, J. G., Brown, K. M., Lynch, J. P. (2014b): Reduced root cortical cell file number improves drought tolerance in maize. - Plant Physiology 166(4): 1943-1955.

[11] Ehdaie, B., Merhaut, D. J., Ahmadian, S., Hoops, A. C., Khuong, T., Layne, A. P., Waines, J. G. (2010): Root system size influences water-nutrient uptake and nitrate leaching potential in wheat. - Journal of Agronomy and Crop Science 196(6): 455-466.

[12] Ekasingh, B., Gypmantasiri, P., Thong-Ngam, K., Grudloyma, P. (2014): Maize in Thailand: Production Systems, Constraints, and Research Priorities -Mexico, D.F. CIMMYT.

[13] Gao, Y., Lynch, J. P. (2016): Reduced crown root number improves water acquisition under water deficit stress in maize (Zea mays L.). - Journal of Experimental Botany 67(15): 76-81.

[14] Garcia, A. A. F., Wang, S., Melchinger, A. E., Zeng, Z.-B. (2008): Quantitative trait loci mapping and the genetic basis of heterosis in maize and rice. - Genetics 180(3): 17071724.

[15] Hochholdinger, F., Park, W. J., Sauer, M., Woll, K. (2004a): From weeds to crops: genetic analysis of root development in cereals. - Trends in Plant Science 9(1): 42-48.

[16] Hochholdinger, F., Woll, K., Sauer, M., Dembinsky, D. (2004b): Genetic dissection of root formation in maize (Zea mays) reveals root-type specific developmental programmes. - Annals of Botany 93(4): 359-368.

[17] Hochholdinger, F., Woll, K., Sauer, M., Feix, G. (2005): Functional genomic tools in support of the genetic analysis of root development in maize (Zea mays L.). - Maydica 50: 437-442.

[18] Hochholdinger, F., Wen, T. J., Zimmermann, R., Chimot-Marolle, P., Da Costa E Silva, O., Bruce, W., Lamkey, K. R., Wienand, U., Schnable, P. S. (2008): The maize (Zea mays L.) roothairless3 gene encodes a putative GPI-anchored, monocot-specific, COBRA-like protein that significantly affects grain yield. - Plant Journal 54(5): 888-898. 
[19] Hoecker, N., Keller, B., Piepho, H.-P., Hochholdinger, F. (2006): Manifestation of heterosis during early maize (Zea mays L.) root development. - Theoretical and Applied Genetics 112(3): 421-429.

[20] Hufford, M. B., Xu, X., van Heerwaarden, J., Pyhäjärvi, T., Chia, J.-M., Cartwright, R. A., Elshire, R. J., Glaubitz, J. C., Guill, K. E., Kaeppler, S. M., Lai, J., Morrell, P. L., Shannon, L. M., Song, C., Springer, N. M., Swanson-Wagner, R. A., Tiffin, P., Wang, J., Zhang, G., Doebley, J., McMullen, M. D., Ware, D., Buckler, E. S., Yang, S., RossIbarra, J. (2012): Comparative population genomics of maize domestication and improvement. - Nature Genetics 44(7): 808-811.

[21] Liu, X., Zhang, S., Shan, L. (2013): Heterosis for water uptake by maize (Zea mays L.) roots under water deficit: responses at cellular, single-root and whole-root system levels. - Journal of Arid Land 5(2): 255-265.

[22] Lynch, J. P. (2005): Root Architecture and Nutrient Acquisition. - BassiriRad, H. Nutrient Acquisition by Plants. Springer-Verlag, Berlin/Heidelberg.

[23] Lynch, J. P. (2007): Roots of the second green revolution. - Australian Journal of Botany 55: 493-512.

[24] Lynch, J. P. (2013): Steep, cheap and deep: an ideotype to optimize water and N acquisition by maize root systems. - Annals of Botany 112(2): 347-357.

[25] Lynch, J. P., Ho, M. D. (2005): Rhizoeconomics: Carbon costs of phosphorus acquisition. - Plant and Soil 269(1-2): 45-56.

[26] Palta, J. A., Chen, X., Milroy, S. P., Rebetzke, G. J., Dreccer, M. F., Watt, M. (2011): Large root systems: are they useful in adapting wheat to dry environments? - Functional Plant Biology 38: 347-354.

[27] Pipitkul, P., Chanakai, S., Kerdpol, P., Limsawad, Y., Wongvidecha, K., Vatchrangkool, T., Suton, N., Rakdech, R., Techakampolsarakij, P., Malaikritsanachalee, K., Changsi, S., Gawga, S., Pitaksenakul, S., Jeengao, N. (2015): Agricultural Statistic of Thailand, 2015 - Buddhapress, Bangkok.

[28] Postma, J. A., Dathe, A., Lynch, J. P., Science, P., Pennsylvania, J. A. P. (2014): The optimal lateral root branching density for maize depends on nitrogen and phosphorus. Plant Physiology 166(2): 590-602.

[29] Qayyum, A., Ahmad, S., Liaqat, S., Malik, W., Noor, E., Muhammad, H. (2012): Screening for drought tolerance in maize (Zea mays L.) hybrids at an early seedling stage. - African Journal of Agricultural Research 7(24): 3594-3603.

[30] R Core Team (2017): R: A language and environment for statistical computing. - R Foundation for Statistical Computing, Vienna, Austria. http://www.r-project.org/. (accessed: 3 July 2017).

[31] Ranum, P., Peña-Rosas, J. P., Garcia-Casal, M. N. (2014): Global maize production, utilization, and consumption. - Annals of the New York Academy of Sciences 1312(1): 105-112.

[32] Reynolds, T. W., Waddington, S. R., Anderson, C. L., Chew, A., True, Z., Cullen, A. (2015): Environmental impacts and constraints associated with the production of major food crops in Sub-Saharan Africa and South Asia. - Food Security 7(4): 795-822.

[33] Richards, R. A. (2006): Physiological traits used in the breeding of new cultivars for water-scarce environments. - Agricultural Water Management 80: 197-211.

[34] Saengwilai, P., Nord, E. A, Chimungu, J. G., Brown, K. M., Lynch, J. P. (2014a): Root cortical aerenchyma enhances nitrogen acquisition from low-nitrogen soils in maize. Plant Physiology 166(2): 726-735.

[35] Saengwilai, P., Tian, X., Lynch, J. P. (2014b): Low crown root number enhances nitrogen acquisition from low-nitrogen soils in maize. - Plant Physiology 166(2): 581-589.

[36] Salvi, S., Giuliani, S., Ricciolini, C., Carraro, N., Maccaferri, M., Presterl, T., Ouzunova, M., Tuberosa, R. (2016): Two major quantitative trait loci controlling the number of seminal roots in maize co-map with the root developmental genes rtcs and rum1.Journal of Experimental Botany 67(4): 1149-1159. 
[37] Schindelin, J., Arganda-Carreras, I., Frise, E., Kaynig, V., Longair, M., Pietzsch, T., Preibisch, S., Rueden, C., Saalfeld, S., Schmid, B., Tinevez, J.-Y., White, D. J., Hartenstein, V., Eliceiri, K., Tomancak, P., Cardona, A. (2013): Fiji-an open source platform for biological image analysis. - Natural Methods 9(7): 1-15.

[38] Springer, N. M., Stupar, R. M. (2007): Allelic variation and heterosis in maize: how do two halves make more than a whole? - Genome research 17(3): 264-275.

[39] Thiemann, A., Fu, J., Seifert, F., Grant-Downton, R. T., Schrag, T. A., Pospisil, H., Frisch, M., Melchinger, A. E., Scholten, S. (2014): Genome-wide meta-analysis of maize heterosis reveals the potential role of additive gene expression at pericentromeric loci. BMC Plant Biology 14: 88.

[40] Thomas, C. L., Graham, N. S., Hayden, R., Meacham, M. C., Neugebauer, K., Nightingale, M., Dupuy, L. X., Hammond, J. P., White, P. J., Broadley, M. R. (2016): High-throughput phenotyping (HTP) identifies seedling root traits linked to variation in seed yield and nutrient capture in field-grown oilseed rape (Brassica napus L.). - Annals of Botany 118(4): 655-665.

[41] Tilman, D., Cassman, K. G., Matson, P. A., Naylor, R., Polasky, S. (2002): Agricultural sustainability and intensive production practices. - Nature 418: 671-677.

[42] Tollenaar, M., Ahmadzadeh, A., Lee, E. A. (2004): Physiological basis of heterosis for grain yield in maize. - Crop Science 44(6): 2086-2094.

[43] Tuberosa, R., Salvi, S. (2009): QTL for Agronomic Traits in Maize Production. - In: Bennetzen, J., Hake, S. (eds.) Handbook of Maize: Its Biology. Springer New York.

[44] Tuberosa, R., Sanguineti, M. C., Landi, P., Giuliani, M. M., Salvi, S., Conti, S. (2002): Identification of QTLs for root characteristics in maize grown in hydroponics and analysis of their overlap with QTLs for grain yield in the field at two water regimes. Plant Molecular Biology 48: 697-712.

[45] York, L. M., Galindo-Castañeda, T., Schussler, J. R., Lynch, J. P. (2015): Evolution of US maize (Zea mays L.) root architectural and anatomical phenes over the past 100 years corresponds to increased tolerance of nitrogen stress. - Journal of Experimental Botany 66(8): 2347-2358.

[46] Zanoni, U., Dudley, J. W. (1989): Comparison of different methods of identifying inbreds useful for improving elite maize hybrids. - Crop Science 29(3): 577.

[47] Zhu, J., Mickelson, S. M., Kaeppler, S. M., Lynch, J. P. (2006): Detection of quantitative trait loci for seminal root traits in maize (Zea mays L.) seedlings grown under differential phosphorus levels. - Theoretical and Applied Genetics 113(1): 1-10.

[48] Zhu, J., Brown, K. M., Lynch, J. P. (2010): Root cortical aerenchyma improves the drought tolerance of maize (Zea mays L.). - Plant, Cell \& Environment 33(5): 740-749. 